\title{
A Novel Transdermal Delivery System for the Anti-Inflammatory Lumiracoxib: Influence of Oleic Acid on In Vitro Percutaneous Absorption and In Vivo Potential Cutaneous Irritation
}

\author{
Tailane Sant'Anna Moreira, ${ }^{1}$ Valéria Pereira de Sousa, ${ }^{1}$ and Maria Bernadete Riemma Pierre ${ }^{1,2}$
}

Received 14 October 2009; accepted 9 March 2010; published online 7 April 2010

\begin{abstract}
Transdermal delivery of non-steroidal anti-inflammatory drugs may be an interesting strategy for delivering these drugs to the diseased site, but it would be ineffective due to low skin permeability. We investigated whether oleic acid (OA), a lipid penetration enhancer in poloxamer gels named poloxamer-based delivery systems (PBDS), can improve lumiracoxib (LM) delivery to/through the skin. The LM partition coefficient $(K)$ studies were carried out in order to evaluate the drug lipophilicity grade ( $K_{\text {octanol/buffer }}$ ), showing values $>1$ which demonstrated its high lipophilicity. Both in vitro percutaneous absorption and skin retention studies of LM were measured in the presence or absence of OA (in different concentrations) in PBDS using porcine ear skin. The flux of in vitro percutaneous absorption and in vitro retention of $\mathrm{LM}$ in viable epidermis increased in the presence of $10.0 \%(w / w)$ OA in $25.0 \%(w / w)$ poloxamer gel. In vivo cutaneous irritation potential was carried out in rabbits showing that this formulation did not provide primary or cumulative cutaneous irritability in animal model. The results showed that $25.0 \%$ poloxamer gel containing $10.0 \% \mathrm{OA}$ is potential transdermal delivery system for LM.
\end{abstract}

KEY WORDS: in vitro skin permeation; lumiracoxib; oleic acid; poloxamer-based delivery systems; skin delivery.

\section{INTRODUCTION}

Lumiracoxib (LM), [2-(2-chloro-6-fluorophenylamino)5-methyl phenyl] acetic acid (Fig. 1), is a new selective nonsteroidal anti-inflammatory (NSAID) cyclooxygenase (COX)-2 inhibitor developed for the management of chronic pain associated with osteoarthritis, rheumatoid arthritis, and acute pain. An increased expression of COX-2 in several cancers has been observed in recent studies which also report the anticancer effects of COX-2 inhibitors in lung cancer, adenocarcinoma of the colon, intestinal polyposis, and even skin cancers $(1,2)$.

The ability of LM in controlling pain and inflammation with reduction of gastrointestinal adverse effects is one of the advantages of its use $(3,4)$. It is suggested that a daily $100-\mathrm{mg}$ oral LM dosage is not associated with an increase of hepatic malfunction compared with other NSAIDs. However, there is a risk of nonlethal liver damage in long-term treatments $(5,6)$. Thus, LM transdermal delivery could be an interesting alternative to the oral route in the treatment of inflammatory or acute pain associated with arthritis and arthrosis because it avoids the occurrence of hepatic side effects associated with

\footnotetext{
${ }^{1}$ School of Pharmacy, Pharmaceutical Technology Laboratory, Department of Medicine, Federal University of Rio de Janeiro, Av. Carlos Chagas Filho, 373, 21.941.590, Rio de Janeiro, Brazil.

${ }^{2}$ To whom correspondence should be addressed. (e-mail: bernadete@ pharma.ufrj.br)
}

systemic delivery. There is no transdermal formulation available for LM delivery, and its penetration properties through human skin have not been investigated. Considering that most inflammatory diseases occur locally and near the body surface, transdermal delivery of NSAID may turn out to be an interesting strategy to deliver these drugs to the deeper layers of the skin in increased concentrations and directly to the diseased site. However, poor skin permeability limits the efficacy of transdermal delivery systems.

The stratum corneum (SC) is considered the main skin barrier limiting the penetration of highly hydrophilic or lipophilic drugs. To optimize the transdermal delivery of these drugs, it is necessary to use techniques that reduce the diffusional resistance of the SC. Many studies have used physical and chemical techniques to disrupt the SC barrier $(7,8)$. Oleic acid $(\mathrm{OA})$ is a cis-unsaturated free fatty acid found abundantly in nature, including in the human skin. Its main mechanism of action is the ability to enhance skin permeability by disorganizing SC intercellular lipid structures (9) without any toxic damage $(10,11)$. Recently, OA has been tested in various in vitro studies as a skin penetration enhancer for many anti-inflammatory (12-16) and also anticancer drugs (17).

Poloxamers are non-ionic polyoxyethylene-polyoxypropilene-polyoxyethylene (PEOn-PPOn-PEOn) tri-block copolymers with many pharmaceutical applications. Poloxamer 127 (Pluronic ${ }^{\circledR}$ F-127 or PF-127) has been the most widely used copolymer in pharmaceutical preparations, mainly in drug delivery due to its biocompatibility; besides, it can form 


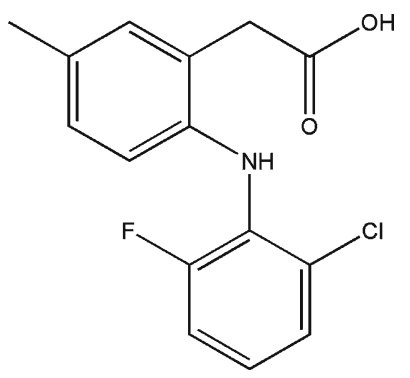

Fig. 1. Chemical structure of lumiracoxib

a gel that provides "depot-like" sustained drug release. Poloxamers has been used as a delivery system of many drugs for ophthalmic $(18,19)$, rectal $(20,21)$, parenteral $(22,23)$, topical $(24)$, and transdermal $(25,26)$ applications. Recently, PF-127 gel has been explored as a skin delivery system to several NSAIDs as naproxen (27), indometacin (28), piroxicam (26), diclofenac (29), among others, due to higher drug retention at the action site which leads to a sustained drug release and improved therapeutic efficiency.

In the present study, we investigated whether poloxamerbased delivery systems (PBDS) containing the skin penetration enhancer (OA) could promote the in vitro percutaneous absorption of LM using porcine ear skin. The potential of LM transdermal delivery to cause primary and cumulative dermal irritations in an animal model in vivo was also investigated.

\section{MATERIAL AND METHODS}

\section{Chemicals}

Poloxamer 127 (Pluronic F-127® or PF-127), oleic acid, and cellulose membranes (MWCO 12,000) were obtained from Sigma Chemical (St Louis, MO, USA). Lumiracoxib was provided by Novartis Pharmaceutics (São Paulo, SP, Brazil). All other chemicals were of analytical grade and were purchased from Merck (Darmstadt, Germany).

\section{HPLC Assay for LM}

The HPLC system consisted of a Shimadzu liquid chromatograph, model SPD M-10AD VP (with a photodiode array detector at $278 \mathrm{~nm}$ ), an LC-10ADVP pump, an autoinjector SIL-10AD VP, and a Class VP integrator software. Two different methods were developed to analyze LM in different samples, as described below.

\section{LM Quantification in Samples from Partition Coefficient (Koctanol/buffer $)$ Studies}

Samples were submitted to HPLC on a propylsulphonic column (J. T. Backer) $250 \times 4.6 \mathrm{~mm}(5 \mu \mathrm{m})$ at room temperature $\left(25^{\circ} \mathrm{C}\right)$, a mobile phase of phosphate buffer $0.1 \mathrm{M}, \mathrm{pH}$ 7.4/water/acetonitrile (100:400:500, v/v/v), $1 \mathrm{~mL} / \mathrm{min}$ flux, and $25 \mu \mathrm{L}$ injection volume. LM retention time was $2.6 \mathrm{~min}$. The assay was linear for concentrations between 10 and $50 \mu \mathrm{g} / \mathrm{mL}$ $(r=0.999)$ with an injection variability of $<1 \%$ for intra-day variation and $<3 \%$ for inter-day variation. Detection limit (DL) was $1.28 \mu \mathrm{g} / \mathrm{mL}$ with a precision of $5 \%$ and the quantification limit (QL) $4.27 \mu \mathrm{g} / \mathrm{mL}$.

\section{LM Quantification in Samples of In Vitro Skin Permeation and Retention Studies}

HPLC was performed on a $\mathrm{C}_{8}$ reverse phase column Schinpack (Shimadzu) $150 \times 4.6 \mathrm{~mm}(5 \mu \mathrm{m})$ at room temperature $\left(25^{\circ} \mathrm{C}\right)$. An acetonitrile/0.01 $\mathrm{M}$ sodium phosphate buffer, pH2.5 $(50 / 50, v / v)$ mixture was used as the mobile phase at a flux rate of $1 \mathrm{~mL} / \mathrm{min}$ and a sample injection volume of $50 \mu \mathrm{l}$. LM retention time was $7.6 \mathrm{~min}$. The assay was linear between concentrations of 4 and $200 \mu \mathrm{g} / \mathrm{mL}$, with a correlation coefficient $(r)$ of 0.9998 and an injection variability of $<1 \%$ for intra-day and $<3 \%$ for inter-day variation. DL was $0.012 \mu \mathrm{g} / \mathrm{mL}$ with a $5 \%$ precision, and the QL of this HPLC assay was $0.041 \mu \mathrm{g} / \mathrm{mL}$. The values obtained are considered suitable for both analytical assays (30). Both HPLC methods were validated during 6 days, and the coefficients of variation for precision and accuracy were below 3\%. Unidentified peaks were not detected in both HPLC procedures.

\section{LM Octanol/Buffer Partition Coefficient ( $\left.K_{\text {octanol/buffer }}\right)$}

Measurements of LM 1-octanol/buffer partition coefficients were carried out using the shake-flask method according to the OECD guideline 107, 1995 (31). LM was dissolved in $0.1 \mathrm{M}$ phosphate buffer ( $\mathrm{pH} 7.4$ ) previously saturated with octanol at a concentration of $60 \mu \mathrm{g} / \mathrm{mL}$ and mixed with the same volume of octanol also previously saturated with phosphate buffer. Samples were stirred for $30 \mathrm{~min}$, centrifuged to separate the two phases, and the amount of LM in the aqueous phase was quantified by HPLC as described above at time zero $\left(C_{0}\right)$ and after shaking to ensure partition $\left(C_{\mathrm{t}}\right)$. The partition coefficient was calculated as $K_{\text {octanol/buffer }}=$ $\left(C_{0}-C_{\mathrm{t}}\right) / C_{\mathrm{t}}(32,33)$.

\section{Preparation of Gels}

Poloxamer-based delivery systems were prepared from PF-127 which forms a gel under hydration by the cold process (34). Concentrations of PF-127, LM, and OA reported here are expressed as percentage of weight/weight $(\%, w / w$; Table I). Appropriate amounts of PF-127 sufficient to yield $20.0 \%, 25.0 \%$, and $30.0 \%$ gels were slowly added to cold water $\left(4-5^{\circ} \mathrm{C}\right)$ under constant stirring to increase the solubilization rate. Dispersions were kept refrigerated until a clear solution was formed. Gels loaded with LM and OA were prepared (Table I) by adding to the cold solution weighed amounts of the drug $(1.0 \%)$ and OA $(1.0 \%, 2.5 \%, 5.0 \%$, and $10.0 \%$ ) previously dispersed in the co-solvent propylene glycol (PG). The final concentration of PG was $20.0 \%(w / w)$ in the poloxamer gels. The solutions were then brought to volume with water and thoroughly stirred while cold. Control gel formulations were represented by $\mathrm{PF}-127$ in all three concentrations containing LM (1.0\%) and $20.0 \%$ PG without OA.

\section{In Vitro Skin Permeation and Retention Studies}

Porcine ears obtained from a local slaughterhouse had the dorsal skin removed from the underlying cartilage with a scalpel (hairs not removed) and were stored wrapped in aluminum foil at $-20^{\circ} \mathrm{C}$ for a maximum of 4 weeks before use, 
Table I. P-127 Gel Formulations and Control with Their Respective OA Concentrations

\begin{tabular}{ccccc}
\hline Samples & $\begin{array}{l}\mathrm{PF}-127 \\
(\%, w / w)\end{array}$ & $\begin{array}{l}\mathrm{OA}^{a} \\
(\%, w / w)\end{array}$ & $\begin{array}{l}\mathrm{LM}^{a} \\
(\%, w / w)\end{array}$ & $\begin{array}{l}\mathrm{PG}^{a} \\
(\%, w / w)\end{array}$ \\
\hline Gel formulations & 20 & 10.0 & 1 & 20 \\
& 25 & 1.0 & 1 & 20 \\
& & 2.5 & 1 & 20 \\
& & 5.0 & 1 & 20 \\
Control gels & 30 & 10.0 & 1 & 20 \\
(without AO) & 20 & - & 1 & 20 \\
& 30 & - & 1 & 20 \\
& 30 & - & 1 & 20 \\
\hline
\end{tabular}

All formulations contain LM $1 \%(w / w)$ and PG as co-solvent $(20.0 \%$, $w / w)$

${ }^{a}$ These percentages, $w / w$, are related to the amounts of these substances $(\mathrm{g})$ for $100 \mathrm{~g}$ of the poloxamer gel formulations or controls

as already described (35). The skin samples were mounted on a diffusion system (Fig. 2) with the SC facing the donor compartment (exposition area $=1.13 \mathrm{~cm}^{2}$ ) and the dermis facing the acceptor solution $(100 \mathrm{~mL}$ phosphate buffer $0.1 \mathrm{M}$, $\mathrm{pH} 7.4$, solution with $3.0 \%$ of polysorbate 20 ). The LM solubility determined in this medium was $340.7 \mu \mathrm{g} / \mathrm{mL}$. In order to ensure sink conditions, the volume of acceptor solution was fixed at $100.0 \mathrm{~mL}$. The sink condition is considered satisfactory if the LM concentration in this medium is at least ten times smaller than the experimental drug solubility of LM, considering that all drugs in the donor compartment were permeated through the skin.

The cold PF-127 gel solutions (0.2 g containing $2 \mathrm{mg}$ drug) were introduced into the donor compartment, and $1 \mathrm{~mL}$ of the acceptor solution (maintained at $37^{\circ} \mathrm{C}$ and stirred with a magnet bar at $500 \mathrm{rpm}$ ) was withdrawn at predetermined time points $(0,0.5,1,2,4,6,8,12$, and $24 \mathrm{~h})$, followed by the reposition of an equal volume of fresh medium. The collected fractions were filtered through a $0.45-\mathrm{mm}$ membrane (filtering unity, Millipore Corporate Headquarters, Billerica, MA, USA) and the filtrates were analyzed by HPLC as described before in order to quantify the amount of LM diffused into the acceptor solution. The cumulative amount of LM permeated through the skin was plotted as a function of time. The concentration of drug in the acceptor solution was an index of transdermal delivery. The flux values $(J)$ or the rate of drug diffusion through the skin as a function of time was calculated from the slopes of the linear portion (steady state) of in vitro permeation curves at the interval of 8-24 h.

Twenty-four hours after permeation, skin samples were withdrawn from the donor compartment, the surfaces thoroughly washed with distilled water to remove excess formulation, and carefully wiped with tissue paper. To determine the amount of LM retained in the SC, the samples were tape-stripped ten times $(17,33,36,37)$ using Scotch Book Tape no. 845 ( 3 M, St. Paul, MN) and the tape strips subsequently immersed in a vial containing $10 \mathrm{~mL}$ of acetonitrile and shaken for $3 \mathrm{~min}$ in order to extract the LM retained in the skin layer. Samples were filtered through a $0.45-\mu \mathrm{m}$ polytetrafluoroethylene (PTFE) membrane (Corning Incorporated, Corning, NY, USA). The remaining skin [Epidermis without SC + Dermis] was cut into small pieces and vortex-mixed for $1 \mathrm{~min}$ in acetonitrile $(5 \mathrm{~mL})$, sonicated in an ultrasound bath (40 kHz, continuous mode) for $30 \mathrm{~min}$ at $25^{\circ} \mathrm{C}$, and filtered through a $0.45-\mu \mathrm{m}$ PTFE membrane. Aliquots of each filtrate, SC and [Epidermis without SC + Dermis] were analyzed by HPLC in order to quantify LM present in these skin layers, respectively. The amount of LM retained in both skin layers was expressed in micrograms per square centimeter. The recovery of LM from the SC and [Epidermis without SC + Dermis] after extraction procedures were $92.5 \%$ and $99.7 \%$, respectively.

\section{Skin Irritation Test}

Skin irritation studies (38) were carried out in rabbits which had free access to standard chow and tap water. The protocols, in accordance to the guidelines of the "Principles of Laboratory Animal Care" were approved by the Committee of Animal Care, Federal University of Rio de Janeiro. Irritation in rabbit skins was visually evaluated after topical application of PF-127 gel containing OA, the formulation that provided the highest in vitro percutaneous absorption and skin retention [Epidermis without $\mathrm{SC}+$ Dermis]. Rabbits weighing about $1.5 \mathrm{~kg}$ were divided into two groups, each

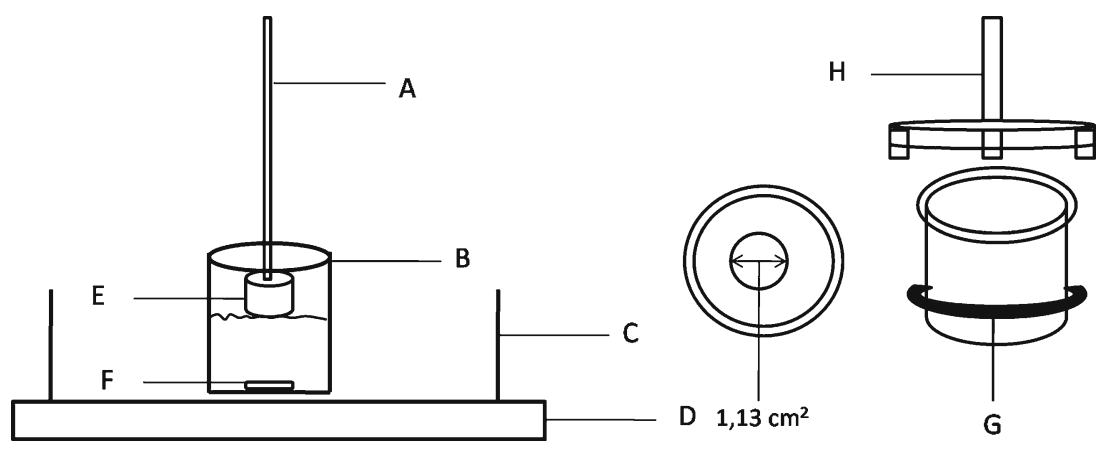

Fig. 2. Diffusion system apparatus: a Support rod fixed in a dissolution system. b Acceptor compartment, $250 \mathrm{~mL}$ vessel. c Thermocontrolled bath. d Multipoint magnetic board. e Donor compartment. f Magnetic bar. $\mathbf{g}$ Teflon elastic. h Attach to the donor compartment attached to the rod 
with three animals, to evaluate primary and cumulative skin irritation. Animal dorsa free of hair were divided into four quadrants of about $4 \mathrm{~cm}^{2}$ each. Two of them were scratched with a needle and the other two remained untouched. About $100 \mathrm{mg}$ of the formulation $(25.0 \%$ poloxamer gel containing $1.0 \% \mathrm{LM}$ and $10.0 \% \mathrm{OA}$ ) was applied occlusively over the dorsum of each animal and covered with gauze and adhesive tape. To evaluate the primary irritation, the first group of treated animals was observed after $4 \mathrm{~h}$. Cumulative irritation was determined by removing the formulation of a second group after $24 \mathrm{~h}$. The dorsum was washed and the formulation was reapplied for five consecutive days, repeating the same procedure described above. The skin visual integrity was evaluated after 24,72 , and $120 \mathrm{~h}$.

\section{Statistical Analysis}

Results are reported as means \pm SEM. Data were statistically analyzed using one-way ANOVA (Tukey's multiple comparisons test). The level of significance was set at $p<0.05$.

\section{RESULTS}

\section{Determinations of the LM Partition Coefficient}

The $K_{\text {octanol/buffer }}$ value for LM was $14.14( \pm 0.83)$, confirming the high lipophilicity of the drug.

\section{In Vitro Permeation Studies}

Figure 3 a shows in vitro LM skin permeation profiles up to $24 \mathrm{~h}$ using formulations containing $1 \% \mathrm{LM}$ and $\mathrm{OA}$ at different concentrations $(1.0 \%, 2.5 \%, 5.0 \%$, and $10.0 \%)$ in PF-127 gels at $25 \%$. Permeation profiles with different slopes can be observed, showing that increases in the OA concentrations from $1.0 \%$ to $10.0 \%$ increases the amount of $\mathrm{LM}$ permeated as a function of time. After $24 \mathrm{~h}$, the amount of LM permeated $\left(\mu \mathrm{g} / \mathrm{cm}^{2}\right)$ through full-thickness porcine skin was significantly higher for PF-127 formulations at $25 \%$ containing $10.0 \%$ OA. Considering $20.0 \%$ and $30.0 \%$ gel concentrations containing only $10.0 \%$ OA (Fig. 3b), the highest amount of LM permeated initially was at $20.0 \%$. However, after $24 \mathrm{~h}$, there was no significant difference between P-127 gel formulations at $20.0 \%$ or $25.0 \%(p<0.05$, Fig. 3a)

LM flux values $(J)$ were calculated from the slopes in the linear portion (steady state, between 8 and $24 \mathrm{~h}$ ) of in vitro permeation curves. Table II shows that among the $25 \% \mathrm{PF}-$ 127 formulations, the flux $J$ increased and the lag time reduced as the OA concentration increased $(p<0.001)$. In these conditions, LM flux through the skin from a $25 \%$ PF127 gel containing OA $10.0 \%$ was approximately 6.5 times higher and the lag time basically the same when compared to control gels at 25\% PF-127 (without OA). Comparing different P-127 gel concentrations $(20.0 \%, 25.0 \%$, and $30.0 \%)$ containing the same concentration of OA $(10.0 \%)$, it is possible to observe that at $30.0 \%$, the flux was significantly reduced $(p<$ 0.001). On the other hand, the lag time increased as the polymer concentration increased. For all samples, a linear relationship was obtained when the total amount of LM in
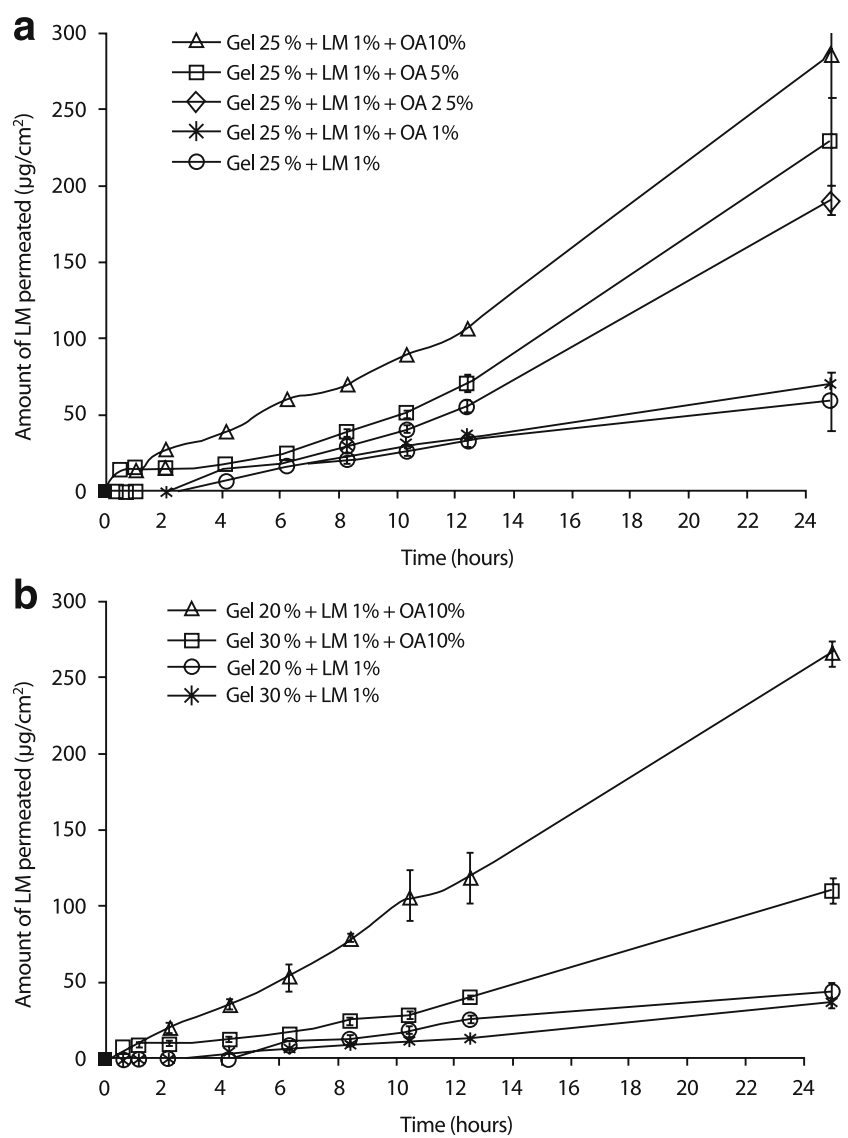

Fig. 3. Cumulative amount of $\mathrm{LM}$ permeated $\left(\mu \mathrm{g} / \mathrm{cm}^{2}\right)$ in vitro through excised porcine skin up to $24 \mathrm{~h}$ from control gels of a PF127 at $25.0 \%$ (without OA) and containing OA (1.0\% 2.5\%, 5.0\%, and $10.0 \%$ ) and b PF-127 at $20.0 \%$ and $30.0 \%$ (without OA) and these gels containing OA $(10.0 \%)$ as function of the time. All formulations contain LM $1 \%$. Each value is the mean of three different experiments \pm SD for each group. Statistical analysis: oneway ANOVA (Tukey's multiple comparison test). Values considered significant $(* * * p<0.001)$ at $24 \mathrm{~h}$ for formulations containing PF-127 at $25.0 \%$ containing OA $1.0 \%, 2.5 \%, 5.0 \%$, and $10.0 \%$ compared to control gel at $25.0 \%$. Among the formulations containing OA $10.0 \%$, there is statistical difference between PF $20 \%$ compared to PF $30.0 \%$ $(* * * p<0.001)$ and PF $25.0 \%$ compared to PF $30.0 \%(* * * p<0.001)$

the receptor solution was plotted against time (zero-order kinetic profile).

\section{In Vitro Retention Studies}

Skin retention studies were carried out to verify the extent of LM retention and its localization in the main skin layers, SC, and [Epidermis without SC + Dermis]. Figure 4a shows values of LM retention both in SC and epidermis + dermis-from PF-127 gels at $25 \%$ with OA concentrations ranging from $1.0 \%$ to $10.0 \%$. The highest amount of LM retained in both SC $\left(14.31 \mu \mathrm{g} / \mathrm{cm}^{2} \pm 0.52\right)$ and [Epidermis without SC + Dermis] $\left(199.1 \mu \mathrm{g} / \mathrm{cm}^{2} \pm 4.26\right)$ was from $25.0 \%$ PF-127 containing $10.0 \%$ OA.

Figure $4 \mathrm{~b}$ shows in vitro retention studies for $20 \%$ and $30 \%$ PF-127 gels containing $10.0 \%$ OA and their respective controls (without OA). The amount of LM retained in SC 
Table II. Values of Flux $J\left(\mu \mathrm{g} / \mathrm{cm}^{2} / \mathrm{h}\right)$, Correlation Coefficient $(r)$, Lag Time (h) After $24 \mathrm{~h}$ of In Vitro Permeation Experiments

\begin{tabular}{lccc}
\hline Poloxamer formulations & Flux $\left(J, \mu \mathrm{g} / \mathrm{cm}^{2} / \mathrm{hour}\right)^{a}$ & Linear correlation $(r)$ & Lag time $(\mathrm{hs}) \pm \mathrm{DP}$ \\
\hline Control gel 25\% & $2.13 \pm 0.98^{b}$ & 0.9898 & $3.06 \pm 0.88$ \\
Gel 25\%+AO 1.0\% & $2.94 \pm 0.37$ & 0.9986 & $6.12 \pm 1.11$ \\
Gel 25\%+AO 2.5\% & $10.43 \pm 0.76$ & 0.9925 & $5.91 \pm 0.50$ \\
Gel 25\%+AO 5.0\% & $12.84 \pm 1.33$ & 0.9935 & $5.58 \pm 0.51$ \\
Gel 25\%+AO 10.0\% & $13.88 \pm 1.61^{c}$ & 0.9941 & $3.50 \pm 0.62$ \\
Control gel 20\% & $1.88 \pm 0.33^{d}$ & 0.9753 & $0.19 \pm 0.64$ \\
Gel 20\%+AO 10.0\% & $11.86 \pm 1.10^{c}$ & 0.9966 & $1.29 \pm 1.72$ \\
Control gel 30\% & $1.78 \pm 0.12^{e}$ & 0.9816 & $3.61 \pm 0.31$ \\
Gel 30\%+AO 10.0\% & $5.68 \pm 0.47^{c}$ & 0.9939 & $4.48 \pm 0.47$ \\
\hline
\end{tabular}

${ }^{a}$ Means \pm SD of the results in three experiments are shown (one-way ANOVA test). Statistical analysis of the flux $(J)$ : one-way ANOVA (multiple comparisons Turkey's test)

${ }^{b}$ Statistically significant for control PF $25 \%$ compared to other gels at $25.0 \%$ containing OA at $2.5 \%, 5.0 \%$ and $10.0 \%(* * * p<0.001)$

${ }^{c}$ Statistically significant $(* * * p<0.001)$ among the formulations containing $10.0 \%$ OA: PF $25 \%$ compared to PF $30 \%$ and PF $20 \%$ compared to PF $30 \%$

${ }^{d}$ Statistically significant $(* * * p<0.001)$ compared with formulation gel PF $20 \%$ containing $10 \%(w / w)$ OA

${ }^{e}$ Statistically significant $(* * * p<0.001)$ compared with formulation gel PF $30 \%$ containing $10 \%(w / w)$ OA

was higher from control gel P-127 at $30.0 \%$. On the other hand, the amount of LM retained in the [Epidermis without $\mathrm{SC}+$ Dermis] was higher in both concentrations when compared to their respective controls $(p<0.001)$ and practically the same for P-127 at $20.0 \%\left(125.06 \pm 3.35 \mu \mathrm{g} / \mathrm{cm}^{2}\right)$ and $30 \%\left(115.22 \pm 2.88 \mu \mathrm{g} / \mathrm{cm}^{2}\right)$ containing $10.0 \%$ OA.

\section{Skin Irritation Test}

The potential skin irritating activity of the previously elected PBDS (PF-127 at 25.0\% containing $10.0 \%$ OA) was analyzed by in vivo evaluation o f the visual appearance of skin rabbits. The assay did not show primary or cumulative
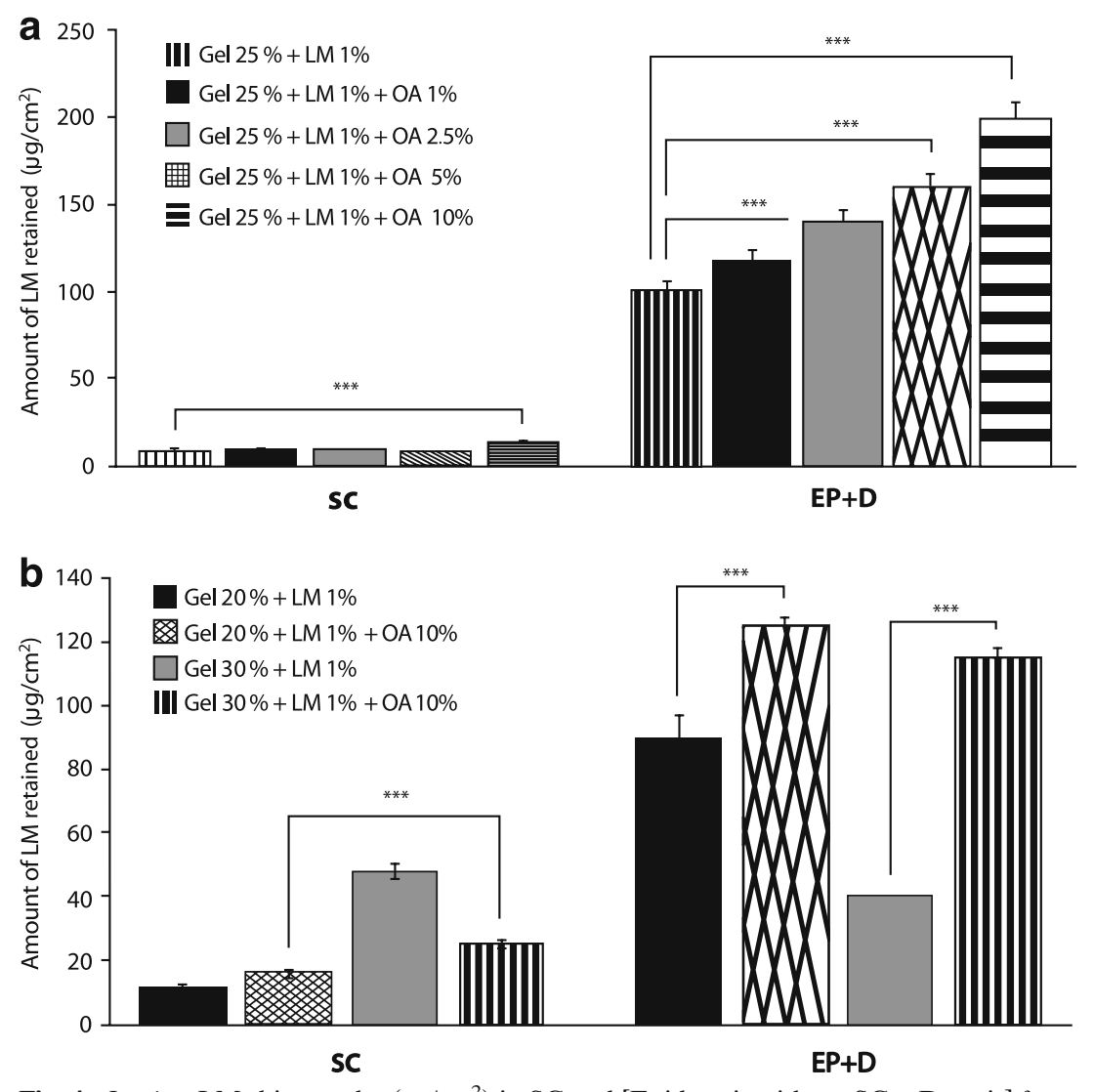

Fig. 4. In vitro $\mathrm{LM}$ skin uptake $\left(\mu \mathrm{g} / \mathrm{cm}^{2}\right)$ in $\mathrm{SC}$ and [Epidermis without $\mathrm{SC}+\mathrm{Dermis}$ ] from control gels and from a PF-127 at $25.0 \%$ (without OA) and containing OA $(1.0 \%, 2.5 \%$, $5.0 \%$, and $10.0 \%$ ) and b PF- 127 at $20.0 \%$ and $30.0 \%$ (without OA) and these gels containing OA $(10.0 \%)$. Each value is the mean of three different experiments \pm SD for each group. Statistical analysis: one-way ANOVA (Tukey's multiple comparison test). Values are considered significant for formulations when $p<0.05(* * * p<0.001)$ 
dermal irritations in the rabbit skin when the formulation was applied for a short period of time $(4 \mathrm{~h})$ or for a longer one (from 24 to $120 \mathrm{~h}$ ), respectively.

\section{DISCUSSION}

The grade of lipophilicity that a drug present in the development of a transdermal delivery system influences its bioavailability. Drugs that show excessive hydrophilicity or lipophilicity do not have an optimal passive transport through the skin, mainly because of the barrier function of the SC. Due to the absence of experimental determinations of these values in the literature, the $K_{\text {octanol/buffer }}$ for LM was evaluated and confirmed the high drug affinity to the nonpolar medium or its high lipophilicity. This value was close to the one described for diclofenac, $K_{\text {Octanol/Buffer }}=13.4$ (39), but the presence of a methyl group instead of hydrogen and fluor instead of chlorine (Fig. 1) slightly increased LM lipophilicity compared to diclofenac.

Several studies have shown the increased percutaneous absorption of anti-inflammatory drugs when oleic acid was present in the transdermal gels (40-43). We carried out the skin permeation studies and assessed the amount of LM permeated, its flux, and lag time, which is the time necessary for the transport of the permeant through the membrane until it reaches equilibrium (44).

In the present paper, the influence of OA concentration on LM permeation across porcine skin showed that the highest concentration of OA $(10.0 \%)$ promoted a higher drug permeation from $25.0 \%$ PF-127 gel (Fig. 3a) and smaller lag time (Table II). This observation seems to be compatible with the main mechanism suggested for the action of OA as a penetration enhancer and shows the direct influence of its concentration on cutaneous permeation: increasing OA concentration increases SC permeability, and consequently, drug diffusion through the membrane is easier $(9,45)$ and the time to reach the steady state is shorter.

Considering the influence of polymer concentration on LM percutaneous absorption, it is possible to observe that gel viscosity increases with polymer concentration (20\% to $30 \%)$, leading to reduced release of the LM from the formulation and consequently the reduction of drug permeation through the skin and increased lag time. Our results (Fig. 3b) show that the gel PF-127 at $30 \%$ containing $10.0 \%$ OA promoted a smaller LM transdermal delivery (across the skin). Linear correlations $(r)$ of the amount of LM permeated $\left(\mu \mathrm{g} / \mathrm{cm}^{2}\right)$ as a function of the time were found for all formulations studied, characterizing a diffusional model. Besides, it indicates that the porcine ear skin is permeable to LM and the permeation profile may be described by zero-order kinetic.

The in vitro permeation studies were carried out using an optimal acceptor medium (phosphate buffer containing polysorbate 20); the surfactant was used in order to increase the LM solubility permeated from this aqueous medium to guarantee sink conditions without apparent damage to the skin. Although topically applied surfactants have the potential to solubilize lipids in the stratum corneum and lead to an increased flow of substances through the skin (46), literature data show that non-ionic surfactants such as polysorbate have less enhancer effect in human skin while ionic surfactants show more pronounced effects (47). Furthermore, the liter- ature has also reported the use of polysorbate 20 as an adjuvant to acceptor media for in vitro experiments $(48,49)$.

Excised animal skin (as porcine ear skin) is frequently used in vitro in percutaneous absorption studies because it is readily accessible in sufficient size and quality compared to human skin. Moreover, this animal model has shown histological and biochemical properties similar to human skin $(50,51)$, including permeability to drugs so that an overestimation of drug penetration into human skin by extrapolation from experiments with porcine skin appears unlikely; that is, an adequate correlation of skin penetration into human and porcine skin can be established (52-54).

Other research groups have shown that porcine skin is particularly suitable for the permeation studies, providing results comparable to human skin $(55,56)$. Thus, it is expected that the flux and retention of LM in human skin should be similar to the results obtained with porcine ear skin in the present study.

PG was used as co-solvent for LM in all gel formulations (tests and controls) before its addition to PBDS. It is known that PG acts as a skin penetration enhancer for several drugs (57-59). Long-chain fatty acids and other hydrophobic enhancers have been applied in combination with a relatively hydrophilic vehicle, such as PG, to achieve an enhanced penetration effect (60). This synergistic enhancement is probably due to the facilitated incorporation of a fatty acid (i.e., oleic acid) into the stratum corneum lipid alkyl domain by the interaction of PG at the polar head group region (61), facilitating the permeation of drugs through the skin (62). As shown in the present paper, the concentration of $\mathrm{PG}$ $(20.0 \%, w / w)$ used in the delivery systems must promote a synergic effect with OA, thus contributing to the increase of the drug permeation flux $(J)$ through the skin. Other studies also reported this synergism between OA-PG with other drugs and carrier systems $(14-16,63,64)$.

It is known that the SC represents the main barrier to drug penetration through the skin, while the epidermis and dermis act as an aqueous gel not showing significant barrier function but strong reservoir effects. According to the literature, the epidermis and dermis are directly related to the flux and percutaneous absorption since these skin layers could be a reservoir to the posterior drug passage to the systemic circulation (65). This fact is particularly interesting in the case of LM because it is an anti-inflammatory drug designed for pain and inflammation treatments, which normally reaches sites as muscles and synovial liquid besides superficial tissues. Moreover, other studies revealed that LM seems to have a higher affinity to the inflamed tissue (3).

Our results showed that the highest LM amount retained was obtained from $25 \%$ PF-127 gel containing 10.0\% OA either in the SC or in [Epidermis without SC + Dermis] (Fig. 4a).

These results are in accordance with the cutaneous permeation experiments in which the formulation PF-127 at $25 \%$ containing $10 \%$ OA promoted the highest permeation of LM through the porcine skin. That is, we can conclude that this OA concentration turns easier the task of LM to overcome the SC barrier, reaching deeper layers of the skin. Consequently, the in vitro percutaneous absorption of LM also increases. Interestingly, Fig. $4 \mathrm{~b}$ shows that the amount of LM retained in the outermost layer of the skin (SC) for the $30 \%$ PF-127control (without OA) was approximately two 
times higher than the same gel containing $10.0 \%$ OA, probably due to the absence of OA which limits LM penetration into the deeper layers of the skin and also because of the higher viscosity of this formulation. Consequently, the LM flux from this formulation was very low (Table II) and the LM released from formulation did not reach the viable epidermis, remaining retained in the SC (due to the absence of $\mathrm{OA}$ ). The amount of $\mathrm{LM}$ retained in [Epidermis without SC + Dermis] was practically the same (around $120 \mu \mathrm{g} / \mathrm{cm}^{2}$ ) between P-127 gels at $20.0 \%$ and $30.0 \%$ containing $10.0 \%$ OA.

OA has been shown as a more efficient enhancer for highly hydrophilic $\left(P_{\text {octanol/water }}<0\right)$ than lipophilic compounds (66), but this was not apparent in the results in this study. Ten percent OA was an efficient penetration enhancer for the highly lipophilic LM probably due to the association with the proposed PBDS. It is known that PF-127 formulations lead to enhanced solubilization of poor water-soluble drugs and a prolonged release profile in many routes of administration. Due to its micellar nature, the PF-127 gels may turn into viscous isotropic liquid crystal gels (67). At low temperatures, the liquid micellar phase is stable and the drug is probably released by diffusion through the extramicellar water channels of the gel matrix (69). The gel cubic structure is induced by increasing the temperature (68). In the present study, LM was incorporated in a PBDS containing $10.0 \%$ $\mathrm{OA}$, which probably becomes an emulsified system containing PF-127 and OA micelles. These last ones could be changing the drug equilibrium in the gel matrix, thus modifying the rate and mechanism of LM release. As a result, association of these delivery systems with OA increases LM retention in the viable epidermis and consequently its percutaneous absorption as a function of time.

Several pharmaceutical preparations contain unsaturated fatty acids (e.g., oleic acid) and PG, but the general use of this combination is sometimes limited due to the high prevalence of dermal side effects, including SC lipid extraction and damage to viable epidermal cells which presumably occurs because of the acidic nature of these fatty acids $(45,70)$. The potential skin irritation by PF-127 at $25.0 \%$ containing $10.0 \%$ OA was investigated due to its higher flux through the skin and higher retention in [Epidermis without SC+Dermis]. No evidence of skin irritation was observed in the experimental animals up to $120 \mathrm{~h}$ ( 5 days), attesting to the safety of this formulation for daily use. Similar results about the potential skin irritation by OA were reported by other research groups (60) showing good skin tolerability in human (71) and rabbit skin in a $72-\mathrm{h}$ trial using $14.9 \%$ OA associated to other penetration enhancers (72). Other studies have shown the synergistic effect of $15.0 \%$ OA mixed with PG as a potential penetration enhancer (14) as well the clinical safety of OAPG for skin application (63).

Our findings are also in accordance with previous studies showing that systems containing $5-10 \%$ OA in propylene glycol as a vehicle did not cause morphological damage to the epidermis when the SC remained intact despite causing increased blood flow measured by laser Doppler velocimetry (LDV) technique (11). This technique measures cutaneous blood flow and consequently evaluates the skin irritation due to topically applied substances. The technique quantifies the degree of irritation compared to visual scoring. The authors evaluated the irritation potential of $\mathrm{PG}$ alone and $\mathrm{PG}$ associated with the AO (0.16 M) and observed that in a 24-h application, AO-PG increased three times the irritation index compared to PG alone. However, the authors observed that although the process does not always produce visible morphological damage (visual changes), it may trigger a reaction of viable skin layers, causing a higher blood flow, which may be assessed by LDV, a useful tool in detecting minor/moderate irritation. In this study, no visual changes related to skin irritation were observed in the animal model (rabbit skin) caused by formulations containing the OA-PG association up to 5 days of application. Other research groups (10) observed that $\mathrm{OA}$ toxicity to the viable epidermal cells was controlled by the SC thickness. The toxic effect can only be observed in the absence of SC (after stripping or in the early growth period of cultured human skin). However, formulations containing OAPG increased the mRNA expression of interleucin 1 or IL-1 pro-inflammatory cytokine (which causes an inflammatory response) after $6 \mathrm{~h}$ of topical application. Since LM is an antiinflammatory drug, it is interesting to speculate that formation of IL-1 would be avoided by the drug which inhibits the cyclooxygenase action, and consequently, the irritation effect of OA is overcome by the drug. This seems a useful additional advantage in a delivery system for cutaneous application.

\section{CONCLUSION}

The present study proposes an innovative strategy for the utilization of LM and emphasizes the potential of the PBDS suggested for this class of drug since no transdermal semi-solid dosage forms for the COX-2 selective drugs are available in the market. The results obtained in this study show that $25.0 \%$ PBDS containing $10.0 \%$ OA optimizes cutaneous delivery of LM to the skin, significantly increasing drug retention of both epidermis and dermis which act as a depot for continuous and gradually percutaneous absorption of the drug. Furthermore, skin irritation tests in rabbits demonstrated that single or multiple applications of the PBDS associated with OA did not cause any visual changes in the skin, like erythema or edema. Finally, it can be concluded that this formulation has the potential to be a transdermal delivery system for LM.

Further bioavailability experiments will be conducted in this animal model in order to elucidate the pharmacokinetic and pharmacodynamic behavior of this transdermal delivery system.

\section{ACKNOWLEDGMENTS}

The authors would like to thank the Fundação de Amparo a Pesquisa do Estado do Rio de Janeiro (FAPERJ) and Comissão de Aperfeiçoamento de Pessoal de Ensino Superior (CAPES), Brazil, for supporting this study. T. S. Moreira, was the recipient of a CAPES fellowship.

\section{REFERENCES}

1. Orengo F, Gerguis J, Phillips R, Guevara A, Lewis AT, Black HS. Celecoxib, a cyclooxygenase-2 inhibitor as a potential chemopreventive to UV-induced skin cancer: a study in the hairless mouse model. Arch Dermatol. 2002;138:751-5. 
2. Afaq F, Adhami VM, Mukhtar H. Photochemoprevention of ultraviolet B signaling and photocarcinogenesis. Mutat Res. 2005;571:153-73.

3. Mysler E. Lumiracoxib (Prexige): a new selective COX-2 inhibitor. Int J Clin Pract. 2004;58:606-11.

4. Tannenbaum H, Berenbaum F, Reginster JY, Zacher J, Robinson J, Poor G et al. Lumiracoxib is effective in the treatment of osteoarthritis of the knee: a 13 week, randomised, double blind study versus placebo and celecoxib. Clin Rheumatol. 2005;25:42-53.

5. Kalbag J, Yeh CM, Milosavljev S, Lasseter K, Oberstein S, Rordorf C. No influence of moderate hepatic impairment on the pharmacokinetics of lumiracoxib, an oral COX-2 selective inhibitor. Pharmacol Res. 2004;50:181-6.

6. Hinz B, Renner B, Cheremina O, Besz D, Zolk O, Brune K. Lumiracoxib inhibits cyclo-oxygenase- 2 completely at the $50 \mathrm{mg}$ dose: is liver toxicity avoidable by adequate dosing? Ann Rheum Dis. 2009;68:289-91.

7. Wang Y, Thakur R, Fan Q, Michniak B. Transdermal iontophoresis: combination strategies to improve transdermal iontophoretic drug delivery. Eur J Pharm Biopharm. 2005;60:179-91.

8. Semalty A, Semalty M, Singh R, Saraf SK, Saraf S. Iontophoretic drug delivery system: a review. Technol Health Care. 2007;15: 237-45.

9. El Maghraby GM, Campbell M, Finnin BC. Mechanisms of action of novel skin penetration enhancers: phospholipid versus skin lipid liposomes. Int J Pharm. 2005;305:90-104.

10. Boelsma E, Tanojo H, Boddé HE, Ponec M. Assessment of the potential irritancy of oleic acid on human skin: evaluation in vitro and in vivo. Toxicol In Vitro. 1996;10:729-42.

11. Tanojo H, Boelsma E, Junginger HE, Ponec M, Bodde HE. In vivo human skin permeability enhancement by oleic acid: a laser Doppler velocimetry study. J Control Rel. 1999;58:97-104.

12. Singh SK, Durrani MJ, Reddy IK, Khan MA. Effect of permeation enhancers on the release of ketoprofen through transdermal drug delivery systems. Pharmazie. 1996;51:741-4.

13. Santoyo S, Ygartua P. Effect of skin pretreatment with fatty acids on percutaneous absorption and skin retention of piroxicam after its topical application. Eur J Pharm Biopharm. 2000;50:24550.

14. Larrucea E, Arellano A, Santoyo S, Ygartua P. Combined effect of oleic acid and propylene glycol on the percutaneous penetration of tenoxicam and its retention in the skin. Eur J Pharm Biopharm. 2001;52:113-9.

15. Gwak HS, Chun IK. Effect of vehicles and penetration enhancers on the in vitro percutaneous absorption of tenoxicam through hairless mouse skin. Int J Pharm. 2002;236:57-64.

16. Kim MJ, Doh HJ, Choi MK, Chung SJ, Shim CK, Kim DD et al. Skin permeation enhancement of diclofenac by fatty acids. Drug Deliv. 2008;15:373-9.

17. Pierre MBR, Ricci Jr E, Tedesco AC, Bentley MVL. Oleic acid as optimizer of the skin delivery of 5-aminolevulinic acid in photodynamic therapy. Pharm Res. 2006;23:360-6.

18. Edsman K, Carlfors J, Petersson R. Rheological evaluation of poloxamer as an in situ gel for ophthalmic use. Eur J Pharm Sci. 1998;6:105-12.

19. Qi H, Chen W, Huang C, Li L, Chen C, Li W et al. Development of a poloxamer analogs/carbopol-based in situ gelling and mucoadhesive ophthalmic delivery system for puerarin. Int $\mathbf{J}$ Pharm. 2007;337:178-87.

20. Barichello JM, Morishita M, Takayama K, Chiba Y, Tokiwa S, Nagai T. Enhanced rectal absorption of insulin-loaded Pluronic $\AA$ F-127 gels containing unsaturated fatty acids. Int J Pharm. 1989;183:125-32.

21. Koffi AA, Agnely F, Ponchel G, Grossiord JL. Modulation of the rheological and mucoadhesive properties of thermosensitive poloxamer-based hydrogels intended for the rectal administration of quinine. Eur J Pharm Sci. 2006;27:328-35.

22. Katakam M, Ravis WR, Banga AK. Controlled release of human growth hormone in rats following parenteral administration of poloxamer gels. J Control Rel. 1997;49:21-6.

23. Veyries ML, Couarraze G, Geiger S, Agnely F, Massias L, Kunzli B et al. Controlled release of vancomycin from Poloxamer 407 gels. Int J Pharm. 1999;192:183-93.

24. Grüning N, Müller-Goymann CC. Physicochemical characterisation of a novel thermogelling formulation for percutaneous penetration of 5-aminolevulinic acid. J Pharm Sci. 2008;97:231123.

25. Miyazaki S, Tobiyama T, Takada M, Attwood D. Percutaneous absorption of indomethacin from pluronic F-127 gels in rats. J Pharm Pharmacol. 1995;47:455-7.

26. Shin SC, Cho CH, Oh IJ. Enhanced efficacy by percutaneous absorption of piroxicam from the poloxamer gel in rats. Int $\mathbf{J}$ Pharm. 2000;17:213-8.

27. Escobar-Chavez JJ, Quintanar-Guerrero D, Ganem-Quintanar A. In vivo skin permeation of sodium naproxen formulated in pluronic F-127 gels: effect of azone and transcutol. Drug Dev Ind Pharm. 2005;31:447-54.

28. Shawesh A, Kallioinens S, Hellen L, Antikainen O. Pluronic F127 gels as a vehicle for topical formulations of indomethacin and rheological behavior of these formulations. Pharmazie. 2002:57:186-90.

29. Yong CS, Yu-Kyoung O, Yong-Il K, Kim JO, Bong-Kyu Y, Jong$\mathrm{Dal} \mathrm{R}$ et al. Physicochemical characterization and in vivo evaluation of poloxamer-based solid suppository containing diclofenac sodium in rats. Int J Pharm. 2005;301:54-61.

30. Causon R. Validation of chromatographic methods in biomedical analysis viewpoint and discussion. J Chromatogr B. 1997;689: $175-80$.

31. OECD guideline for testing of chemicals no. 107. Partition coefficient ( $n$-octanol/water): shake-flask method. OECD, 2005. http://www.oecd.org/dataoecd/17/35/1948169.pdf. Accessed 20 May 2006.

32. Lopez RFV, Collett JH, Bentley MVL. Influence of cyclodextrin complexation on the in vitro permeation and skin metabolism of dexamethasone. Int J Pharm. 2000;200:127-32.

33. De Rosa FS, Tedesco AC, Lopez RFV, Pierre MBR, Lange N, Marchetti JM et al. In vitro skin permeation and retention of 5aminolevulinic acid ester derivatives for photodynamic therapy. J Control Rel. 2003;89:261-9.

34. Schmolka IR. Artificial skin. I. Preparation and properties of pluronic F-127 gels for treatment of burns. J Biomed Mater Res. 1972;6:571-82.

35. Harrison SM, Barry BW, Dugard PH. Effect of freezing on human skin permeability. J Pharm Pharmacol. 1984;35:261-2.

36. Steluti R, De Rosa FS, Collett J, Tedesco AC, Bentley MV. Topical glycerol monooleate/propylene glycol formulations enhance 5-aminolevulinic acid in vitro skin delivery and in vivo protophorphyrin IX accumulation in hairless mouse skin. Eur J Pharm Biopharm. 2005;60:439-44.

37. De Paula D, Martins CA, Bentley MV. Development and validation of HPLC method for imiquimod determination in skin penetration studies. Biomed Chromatogr. 2008;22:1416-23.

38. Draize JH, Woodard G, Calvery HO. Methods for the study of irritation and toxicity of substances applied topically to the skin and mucous membranes. J Pharmacol Exp Ther. 1944;82:37790.

39. The Merck Index. An encyclopedia of chemicals, drugs, and biologicals, 13th ed. Whitehouse Station: Merck Research Laboratories; 2001.

40. Güngör S, Bergişadi N. Effect of penetration enhancers on in vitro percutaneous penetration of nimesulide through rat skin. Pharmazie. 2004;59:39-41.

41. Dubey R, Bommagani M, Venkateswarlu V, Mullangi R, Karnati HR, Thammera RK et al. Ketorolac tromethamine transdermal gel: development, in vitro and in vivo evaluation. J Pain Palliat Care Pharmacother. 2009;23:26-34.

42. Patel NA, Patel NJ, Patel RP. Design and evaluation of transdermal drug delivery system for curcumin as an anti-inflammatory drug. Drug Dev Ind Pharm. 2009;35:234-42.

43. Singh S, Gajra B, Rawat M, Muthu MS. Enhanced transdermal delivery of ketoprofen from bioadhesive gels. Pak J Pharm Sci. 2009;22:193-8.

44. Aulton ME. Delineamento de formas farmacêuticas. 2nd ed. Porto Alegre: Artmed; 2005. p. 513-31.

45. Touitou E, Godin B, Karl Y, Bujanover S, Becker Y. Oleic acid, a skin penetration enhancer, affects Langerhans cells and corneocytes. J Control Rel. 2002;80:1-7.

46. Asbill CS, Michniak BB. Percutaneous penetration enhancers: local versus transdermal activity. Pharm Sci Technol Today. 2000;3:36-41. 
47. Williams AC, Barry BW. Penetration enhancers. Adv Drug Deliv Rev. 2004;56:6003-18.

48. Casagrande R, Georgetti SR, Verri Jr WA, Borin MF, Lopez RFV, Fonseca MJF. In vitro evaluation of quercetin cutaneous absorption from topical formulations and its functional stability by antioxidant activity. Int J Pharm. 2007;328:183-90.

49. Vicentini FTMC, Simi TRM, Del Ciampo JO, Wolga NO, Pitol $\mathrm{DL}$, Iyomasa MM et al. Quercetin in w/o microemulsion: in vitro and in vivo skin penetration and efficacy against UVB-induced skin damages evaluated in vivo. Eur J Pharm Biopharm. 2008; 69:948-57.

50. Meyer W, Schwarz R, Neurand K. The skin of domestic mammals as a model for the human skin, with special reference to the domestic pig. Curr Probl Dermatol. 1978;7:39-52.

51. Meyer W, Kaczab J, Zschemischc NH, Godynickid S, Seegerb J. Observations on the actual structural conditions in the stratum superficiale dermidis of porcine ear skin, with special reference to its use as model for human skin. Ann Anat. 2007;189:143-15.

52. Hawkins GS, Reifenrath WG. Influence of skinsource, penetration cell fluid, and partition coefficient on in vitro skin penetration. J Pharm Sci. 1986;75:378-81.

53. Dick IP, Scott RC. Pig ear skin as an in-vitro model for human skin permeability. J Pharm Pharmacol. 1992;44:640-5.

54. Schmook FP, Meingassner JG, Billich A. Comparison of human skin or epidermis models with human and animal skin in in-vitro percutaneous absorption. Int J Pharm. 2001;215:51-6.

55. Hoeck U, Orup Jacobsen L, Kreilgard B. Comparision of pig skin and human skin for in vitro percutaneous study of morphine prodrug. Proc Int Symp Control Release Bioact Mater. 1994;21: 425-6.

56. Fujii M, Yamanouchi S, Hori N, Iwanaga N, Kawaguchi N, Matsumoto N. Evaluation of Yucatan micropig skin for use as an in vitro model for skin permeation study. Biol Pharm Bull. 1997;20:249-54.

57. Potts RO, Golden GM, Francoeur ML, Mak VHW, Guy RH. Mechanism and enhancement of solute transport across the stratum corneum. J Control Rel. 1991;15:249-60.

58. Bendas B, Schmalfuss U, Neubert R. Influence of propylene glycol as cosolvent on mechanisms of drug transport from hydrogels. Int J Pharm. 1995;116:19-30.

59. Loftsson T, Petersen DS, Le Goffic F, OLafsson JH. Unsaturated glycerol monoethers as novel skin penetration enhancers. Pharmazie. 1997;52:463.
60. Ben-Shabat S, Baruch N, Sintov AC. Conjugates of unsaturated fatty acids with propylene glycol as potentially less-irritant skin penetration enhancers. Drug Dev Ind Pharm. 2007;33:1169-75.

61. Oh SY, Jeong SY, Park TG, Lee JH. Enhanced transdermal delivery of AZT (zidovudine) using iontophoresis and penetration enhancer. J Control Rel. 1998;51:161-8.

62. Squillante E, Needham T, Maniar A, Kislalioglu S, Zia H. Codiffusion of propylene glycol and dimethyl isosorbide in hairless mouse skin. Eur J Pharm Biopharm. 1998;46:265-71.

63. Jiang J, Wang RK. Comparing the synergistic effects of oleic acid and dimethyl sulfoxide as vehicles for optical clearing of skin tissue in vitro. Phys Med Biol. 2004;49:5283-94.

64. Kogan A, Garti N. Microemulsions as transdermal drug delivery vehicles. Adv Colloid Interface Sci. 2006;123-126:369-85.

65. Sing P, Roberts MS. Skin permeability and local tissue concentration of nonsteroidal anti-inflammatory drugs after topical application. J Pharmacol Exp Ther. 1994;286:144-51.

66. Tanojo H. Fatty acids as enhancers of drug permeation across human skin. An integrated in vitro/in vivo study. $\mathrm{PhD}$ thesis, Leiden University, Leiden, The Netherlands, 1996; p. 90-9.

67. Attwood D, Collett JH, Tait CJ. The micellar properties of the poly(oxyethylene)poly(oxypropylene) copolymer Pluronic F 127 in water and electrolyte solution. Int J Pharm. 1985;26:25-33.

68. Escobar-Chávez JJ, López-Cervantes M, Naïk A, Kali YN, Quintanar-Guerrero D, Ganem-Quintanar A. Applications of thermo-reversible Pluronic F-127 gels in pharmaceutical formulations. J Pharm Sci. 2006;9:339-58.

69. Bentley MV, Marchetti JM, Ricardo N, Ali-Abi Z, Collett JH. Influence of lecithin on some physical chemical properties of poloxamer gels: rheological, microscopic and in vitro permeation studies. Int J Pharm. 1999;193:49-55.

70. Sintov A, Ze'evi A, Uzan R, Nyska A. Influence of pharmaceutical gel vehicles containing oleic/sodium oleate combinations on hairless mouse skin, a histological evaluation. Eur J Pharm Biopharm. 1999;47:299-303.

71. Paolino D, Ventura CA, Nisticò S, Puglisi G, Fresta M. Lecithin microemulsions for the topical administration of ketoprofen: percutaneous adsorption through human skin and in vivo human skin tolerability. Int J Pharm. 2002;244:21-31.

72. Escribano E, Calpena AC, Queralt J, Obach R, Doménech J. Assessment of diclofenac permeation with different formulations: anti-inflammatory study of a selected formula. Eur J Pharm Sci. 2003;19:203-10. 